\section{Case Reports in Ophthalmology}

Case Rep Ophthalmol 2018;9:70-75

\title{
Periorbital Necrobiotic Xanthogranuloma Successfully Treated with Intravenous Immunoglobulin
}

\author{
Rose M. Olson ${ }^{a} \quad$ Andrew R. Harrison ${ }^{b, c}$ Amanda Maltry ${ }^{b}$ \\ Ali Mokhtarzadeh ${ }^{\mathrm{b}}$ \\ ${ }^{a}$ University of Minnesota School of Medicine, Minneapolis, MN, USA; ${ }^{b}$ Department of \\ Ophthalmology and Visual Neurosciences, University of Minnesota, \\ Minneapolis, MN, USA; ${ }^{\mathrm{C}}$ Department of Ophthalmology and Otolaryngology, University of \\ Minnesota, Minneapolis, MN, USA
}

\section{Keywords}

Necrobiotic xanthogranuloma · Non-Langerhans histiocytosis · Intravenous immunoglobulin

\begin{abstract}
Background: Necrobiotic xanthogranuloma (NXG) is a rare non-Langerhans histiocytosis with cutaneous manifestations, most commonly of the periorbital skin, and is often associated with hematologic disorders such as monoclonal gammopathy. Treatment of NXG is notoriously difficult, and fraught with recurrence and progression. Case Presentation: The authors describe a case of NXG with periorbital involvement in a patient with a complex autoimmune and hematologic medical history. The biopsy of this rare lesion prompted subsequent evaluation for an underlying disorder, which led to the diagnosis of multiple myeloma. Her NXG lesions demonstrated remarkable clinical improvement after treatment with intravenous immunoglobulin (IVIG). Conclusions: This case demonstrates the ophthalmologist's critical role in the diagnosis and management of NXG, as early detection cannot only prevent ophthalmic consequences such as ocular perforation and blindness, but also prompt further investigation that may reveal an underlying disorder or systemic involvement, including hematologic malignancy as in this case. NXG has been effectively treated with IVIG in a handful
\end{abstract}


of reported cases. To the author's knowledge, this is the third case of periorbital NXG successfully treated with IVIG, and the first in the ophthalmic literature.

(C) 2018 The Author(s)

Published by S. Karger AG, Basel

\section{Background}

Necrobiotic xanthogranuloma (NXG) is a rare chronic granulomatous disease first described by Kossard and Winkelman in 1980 [1, 2]. It typically affects adults in their 60s, with no gender preference [3]. All patients exhibit cutaneous involvement, most commonly of the periorbital skin. Half of the patients will have ophthalmic complaints that may mimic uveitis or scleritis $[4,5]$. Orbital signs can include proptosis, blepharoptosis, and restricted extraocular motility [6].

An important association of NXG is that most patients have or will be diagnosed with an underlying paraproteinemia, particularly IgG kappa light-chain monoclonal gammopathy, which may progress to multiple myeloma [7]. As in our patient, other biological markers associated with NXG include leukopenia in one-third to one-half of patients and cryoglobulinemia $[3,4,7,8]$. The pathogenesis of NXG and its link to paraproteinemias has yet to be elucidated, though many hypotheses have been proposed. NXG has been reported to affect a wide variety of organs, including cardiac, pulmonary, splenic, renal, and facial nerve tissues [7]. Hematologic disorders may precede or follow NXG, and given the high association, patients with NXG require regular follow-up to screen for disease development. No characteristics of NXG have been found to predict disease course or severity. Therefore, with any new diagnosis of NXG, it is recommended to undergo evaluation for systemic involvement. As was the case in our patient, diagnosis of NXG may be critical to early detection of occult malignancy.

Treatment of NXG is challenging due to its rarity and resultant limited data. No controlled trials have been conducted to evaluate efficacy. Many different treatment options have been attempted, with no clear standard treatment established. Thus, choice of treatment is largely decided by patient factors such as disease extent, comorbidities, and tolerability. In patients with NXG associated with an underlying malignancy, treatment should be directed at treatment of that malignancy [9]. Otherwise, common agents used are alkylating agents and systemic glucocorticoids [9]. Other reported modalities include intralesional corticosteroids, interferon alpha, plasmapheresis, extracorporeal photopheresis, laser therapy, radiotherapy, and psoralen plus ultraviolet A photochemotherapy [9]. Surgical debulking has been discouraged due to the high rate of recurrence [6].

High-dose intravenous immunoglobulin (IVIG) has diverse immunoregulatory effects and multiple mechanisms of action. It is beneficial in a variety of autoimmune and inflammatory disorders, due to its anti-inflammatory and immunosuppressive effects via modulation of IgG levels, production of cytokines, effect on lymphocyte function, inhibition of complement deposition, and removal of pathogenic IgG [10]. Though expensive, IVIG has shown rapid clinical results with near-complete remission and few side effects in a handful of reported cases [11-15]. 


\section{Case Reports in Ophthalmology}

Case Rep Ophthalmol 2018;9:70-75

\begin{tabular}{l|c|}
\hline DOI: $10.1159 / 000485913$ & $\odot 2018$ The Author(s). Published by S. Karger AG, Basel \\
\hline
\end{tabular} www.karger.com/cop

Olson et al.: Periorbital Necrobiotic Xanthogranuloma Successfully Treated with Intravenous Immunoglobulin

\section{Case Presentation}

A 44-year-old Brazilian female presented with a 2-year history of worsening bilateral periorbital discoloration, pain, and swelling. Examination revealed yellow, firm papules coalescing into plaques causing cicatricial lagophthalmos, with surrounding erythema, and bilateral periorbital edema (Fig. 1). Similar extensions were noted to the right temple, left chin, and dorsal right forearm. Magnetic resonance imaging with gadolinium of the orbit revealed no enhancement or mass within the orbit. Visual acuity, pupils, and extraocular motility were normal. Biopsy of the right lower eyelid and right temple demonstrated a dermal histiocytic infiltrate surrounding areas of collagen degeneration (necrobiosis). Numerous Touton and foreign-body type multinucleated giant cells were present, consistent with NXG (Fig. 2).

At the time of diagnosis, the patient was being regularly followed by hematology and oncology for a 7-year history of persistent leukopenia thought to be secondary to an uncharacterized autoimmune disorder, as well as history of monoclonal gammopathy of undetermined significance, cryoglobulinemia, and hypocomplementemia. Diagnosis of NXG prompted bone marrow biopsy and aspirate, and CT/PET scan of the chest, abdomen, and pelvis to rule out development of myeloma or occult lymphoma. Serum protein electrophoresis revealed an IgG kappa monoclonal protein spike. Recent hemoglobin was $9.1 \mathrm{~g} / \mathrm{dL}$, felt to be related to the underlying plasma cell disorder. She was normocalcemic and had normal renal function. Her skeletal survey showed no osteolytic lesions. CT scan showed splenomegaly. Finally, bone marrow biopsy revealed 20-30\% IgG kappa monotypic plasma cells on bilateral trephine biopsies. Diagnosis of multiple myeloma was then made and she was treated with CyBorD (cyclophosphamide, bortezomib, and dexamethasone) with subcutaneous bortezomib.

After completing and tolerating 7 cycles of CyBorD without complication, therapy was re-evaluated. The plasma cell myeloma had responded to chemotherapy with a diminution in serum protein and marrow evidence of myeloma, however, an abnormal B cell clonality was still evident. It was decided to complete cycle 8 of CyBorD and only treat further if there was significant acceleration or evolution of her disease, as CyBorD appeared to have reached the major therapeutic benefit, and because of her low marrow cellularity and other medical comorbidities. There was also concern that continuing CyBorD may promote toxicity. After completing cycle 8 of CyBorD, bone marrow aspirate/biopsy and bone survey was obtained. Because there were no significant changes, and IgG kappa monoclonal protein remained stable at $0.4 \mathrm{~g} / \mathrm{dL}$, it was opted to withhold treatment of the asymptomatic myeloma.

During the year of aggressive CyBorD chemotherapy regimen for treatment of myeloma, she had progression of her periocular NXG. Alternative treatment of her NXG eye lesions was then considered, and IVIG was selected due to reports of its success in treating this disfiguring and painful disorder associated with myeloma. No local therapy was tried. She was started on IVIG $0.5 \mathrm{gm} / \mathrm{kg} /$ day for 4 consecutive days administered every 4 weeks with the intent to complete at least 3 cycles of IVIG, and more if it appeared helpful [11]. After 3 cycles of IVIG, the patient noticed improvement in periorbital discoloration and pain.

After 9 cycles, there was dramatic improvement in the appearance of the soft tissue around her eyes. By cycle 16, all previously noted lesions were resolved. She continues to receive monthly injections of IVIG with favorable results at 4 years of follow-up (Fig. 3). Since completion of the initial 8 cycles of CyBorD, her multiple myeloma has been routinely monitored and there has been no significant changes in the objective markers of myeloma off chemotherapy. 


\section{Discussion and Conclusions}

NXG is a progressive, systemic disease that often presents with ocular complaints. This case demonstrates the clinical significance of early diagnosis and management of NXG, as detection cannot only prevent ophthalmic consequences such as ocular perforation and blindness, but also prompt the detection of systemic effects, including hematologic malignancy. The lack of ophthalmic literature on NXG may signify that NXG is underrecognized by the ophthalmologic community. Despite this, the ophthalmologist plays a critical role in the diagnosis, as NXG most commonly presents on the periorbital skin. This case documents periorbital NXG that prompted the detection of an underlying asymptomatic malignancy, as well as successful treatment with IVIG, a promising, yet underutilized approach. To the best of our knowledge, this represents the first case of periorbital NXG successfully treated with IVIG in the ophthalmic literature, with two prior cases of eyelid involvement in the dermatology literature $[14,15]$.

\section{Statement of Ethics}

We have obtained informed consent from the participant to report individual patient data, including images.

\section{Disclosure Statement}

None of the authors have any competing interests.

\section{Funding Sources}

This study was supported by an unrestricted grant from the Research to Prevent Blindness (New York, NY, USA), and the Lions and Lionesses of Minnesota.

\section{Author Contributions}

R.M.O. prepared the first draft of the paper based on input from A.M. A.R.H. oversaw the writing process and was involved in revision. A.M. performed the ocular pathologic analysis and was involved in manuscript revision. A.M. oversaw the paper from the first draft to submission and assisted in multiple revisions.

\section{References}

1 Kossard S, Winkelmann RK: Necrobiotic xanthogranuloma with paraproteinemia. J Am Acad Dermatol 1980;3:257-270.

2 Kossard S, Winkelmann RK: Necrobiotic xanthogranuloma. Australas J Dermatol 1980;21:85-88.

-3 Finan MC, Winkelmann RK: Necrobiotic xanthogranuloma with paraproteinemia. A review of 22 cases. Medicine (Baltimore) 1986;65:376-388.

4 Mehregan DA, Winkelmann RK: Necrobiotic xanthogranuloma. Arch Dermatol 1992;128:94-100. 


\section{Case Reports in Ophthalmology}

(C) 2018 The Author(s). Published by S. Karger AG, Basel www.karger.com/cop

Olson et al.: Periorbital Necrobiotic Xanthogranuloma Successfully Treated with Intravenous Immunoglobulin

5 Robertson DM, Winkelmann RK: Ophthalmic features of necrobiotic xanthogranuloma with paraproteinemia. Am J Ophthalmol 1984;97:173-183.

-6 Ugurlu S, Bartley GB, Gibson LE: Necrobiotic xanthogranuloma: long-term outcome of ocular and systemic involvement. Am J Ophthalmol 2000;129:651-657.

7 Szalat R, Arnulf B, Karlin L, et al: Pathogenesis and treatment of xanthomatosis associated with monoclonal gammopathy. Blood 2011;118:3777-3784.

-8 Spicknall KE, Mehregan DA: Necrobiotic xanthogranuloma. Int J Dermatol 2009;48:1-10.

$>9$ Miguel D, Lukacs J, Illing T, Elsner P: Treatment of necrobiotic xanthogranuloma - a systematic review. J Eur Acad Dermatol Venereol 2017;31:221-235.

10 Ballow M: The IgG molecule as a biological immune response modifier: mechanisms of action of intravenous immune serum globulin in autoimmune and inflammatory disorders. J Allergy Clin Immunol 2011;127:315-323.

11 Hallermann C, Tittelbach J, Norgauer J, Ziemer M: Successful treatment of necrobiotic xanthogranuloma with intravenous immunoglobulin. Arch Dermatol 2010;146:957-960.

12 Rubinstein A, Wolf DJ, Granstein RD: Successful treatment of necrobiotic xanthogranuloma with intravenous immunoglobulin. J Cutan Med Surg 2013;17:347-350.

13 Liszewski W, Wisniewski JD, Safah H, Boh EE: Treatment of refractory necrobiotic xanthogranulomas with extracorporeal photopheresis and intravenous immunoglobulin. Dermatol Ther 2014;27:268271.

14 Pedrosa AF, Ferreira 0, Calistru A, et al: Necrobiotic xanthogranuloma with giant cell hepatitis, successfully treated with intravenous immunoglobulins. Dermatol Ther 2015;28:68-70.

$\$ 15$ Lukács J, Goetze S, Elsner P: Periocular necrobiotic xanthogranuloma successfully treated with intravenous immunoglobulin. Acta Derm Venereol 2017;97:754-755.

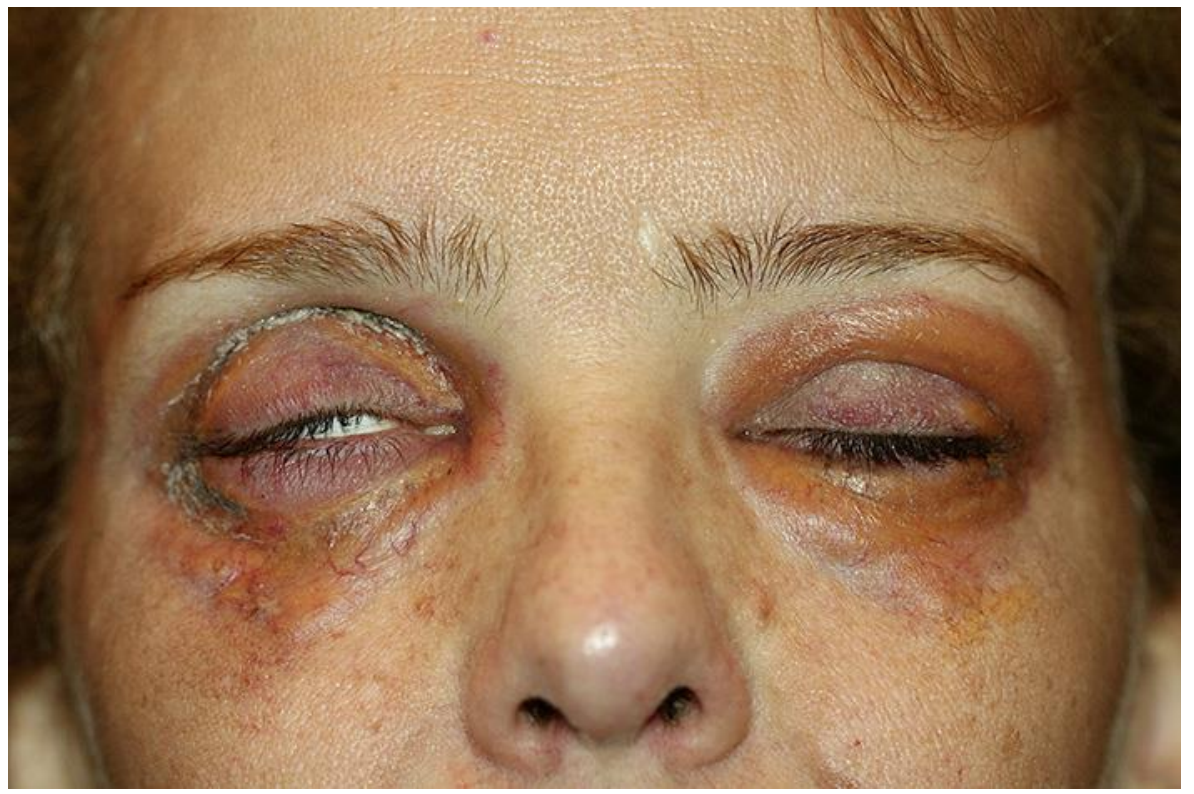

Fig. 1. Pretreatment clinical findings of periorbital necrobiotic xanthogranuloma demonstrating multiple yellow, firm papules coalescing into plaques and resulting in cicatricial lagophthalmos. 


\section{Case Reports in Ophthalmology}

\begin{tabular}{l|l}
\hline Case Rep Ophthalmol 2018;9:70-75 \\
\hline DOI: 10.1159/000485913 & $\begin{array}{l}\text { C 2018 The Author(s). Published by S. Karger AG, Basel } \\
\text { www.karger.com/cop }\end{array}$ \\
\hline
\end{tabular}

Olson et al.: Periorbital Necrobiotic Xanthogranuloma Successfully Treated with Intravenous Immunoglobulin

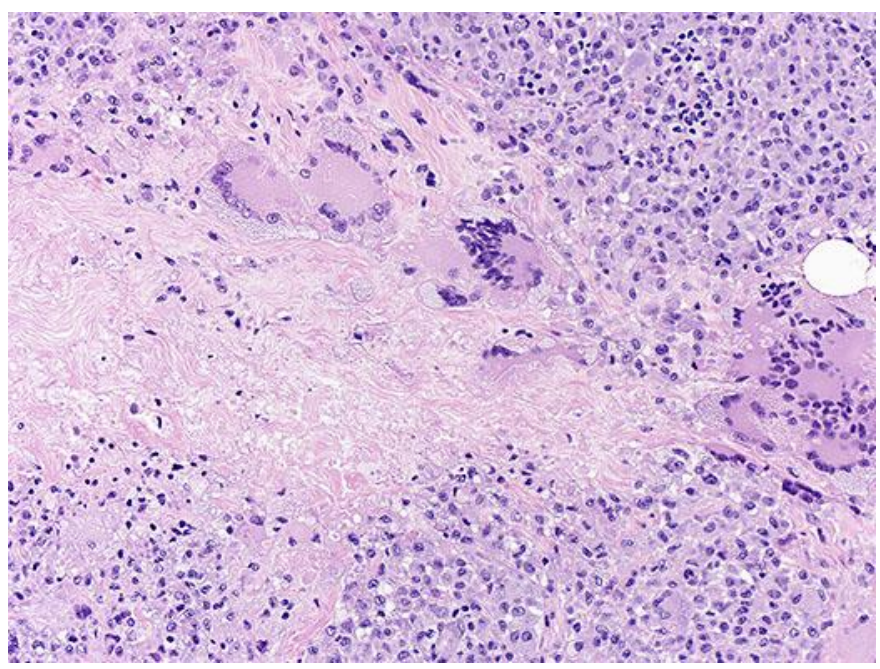

Fig. 2. Skin biopsy of necrobiotic xanthogranuloma lesions revealing a diffuse dermal infiltrate of histiocytes with multinucleated giant cells along with cholesterol clefts, Touton forms, and geographic necrobiosis. Hematoxylin and eosin, $\times 100$.

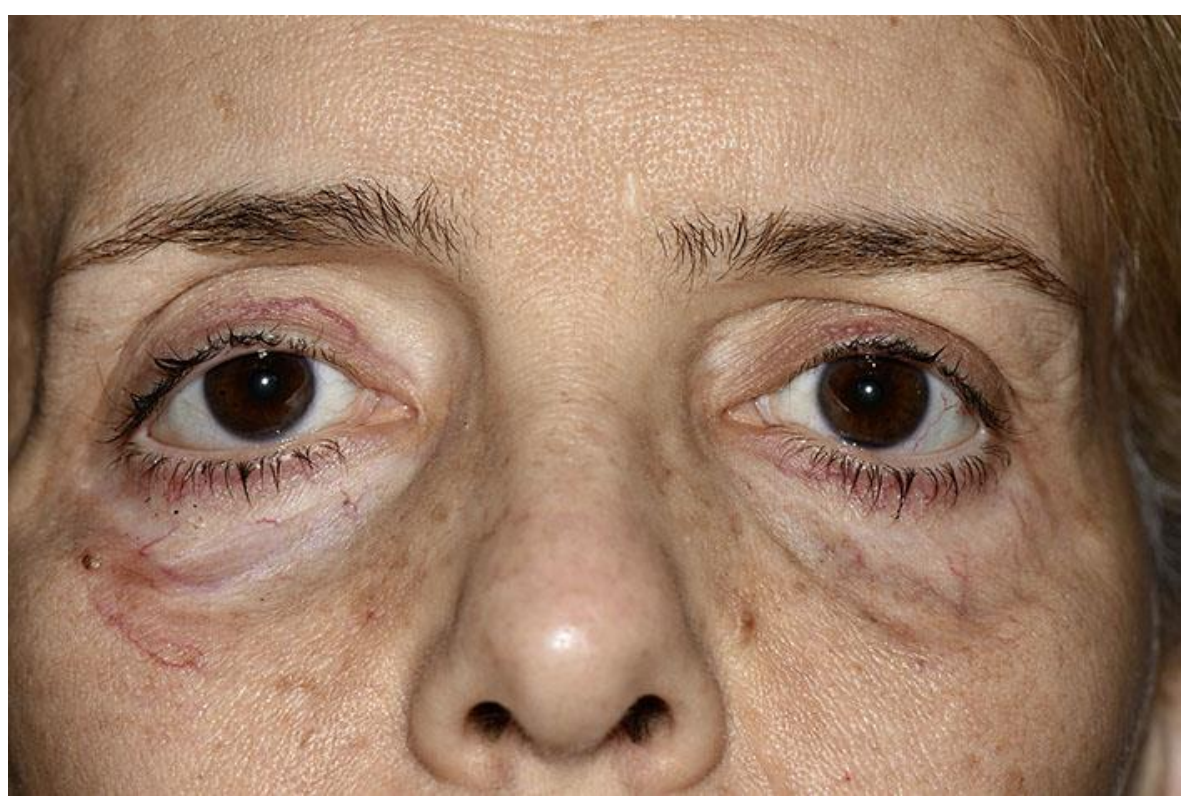

Fig. 3. Treatment outcome of periorbital lesions of necrobiotic xanthogranuloma after 16 cycles of intravenous immunoglobulin. 